\title{
Stress et douleur : conséquences neurophysiologiques. Doit-on contenir nos émotions?
}

\author{
Vianney Descroix \\ UFR d'Odontologie Université Paris Diderot, France \\ Service d'Odontologie Groupe hospitalier Pitié Salpetrière, Paris, France \\ vianney.descroix@psl.aphp.fr
}

Rien n'est moins trivial que la définition d'une émotion. Elle n'est pas passion, ni humeur ou sentiment. Elle est une réponse brève, intense, comportementale et physiologique. Absolument nécessaire à l'existence humaine, elle est aussi bien positive que négative. Parmi ces dernières, le stress, la peur, l'angoisse, l'anxiété ou encore la douleur sont autant d'émotions fréquentes et banales qu'il n'est pas rare d'avoir déjà ressenti et vécu. Elles sont aussi communes que complexes, très souvent intriquées et entremêlées. Peur et douleur sont si intimement liées qu'elles sont indifféremment utilisées et, souvent malencontreusement, on entend encore dire «n'ayez pas peur ça ne va pas faire mal ». La preuve que la douleur fait peur mais aussi que sans doute la peur fait mal... D'ailleurs cela est tellement vrai que différents travaux récents confirment l'existence de voies neuronales et de mécanismes cérébraux communs entre les voies de la douleur et celles des émotions.

Les données actuelles de la science mettent clairement en évidence qu'il se cache derrière ces différentes émotions de véritables enjeux tout à la fois biologiques, médicaux et psychologiques. De nombreux travaux de recherche permettent de comprendre de mieux en mieux les substrats biologiques qui conduisent aux émotions et à leur genèse, mais aussi leur expression et leur ressenti. De la même façon, les conséquences neurologiques, hormonales et psychiques des phénomènes émotionnels ont été clairement décrites. Parce que l'Homme soumis à une agression, à une menace de son homéostasie doit s'adapter, il engage alors un ensemble de réponses biologiques hormonale, immunitaire, cardiovasculaire et cellulaire qui tendent toutes à rétablir un équilibre nécessaire à sa survie, à la fuite ou au combat.

$\mathrm{Au}$ cours de cette conférence, nous aurons à cœur d'interroger les dernières avancées scientifiques permettant une meilleure connaissance des expériences émotionnelles et d'examiner en quoi leurs différentes répercussions sont indispensables à reconnaitre et sans doute peut être à prendre en charge. 